\title{
The current state of implementation science in genomic medicine: opportunities for improvement
}

\author{
Megan C. Roberts, PhD ${ }^{1}$, Amy E. Kennedy, PhD, MPH ${ }^{1}$, David A. Chambers, DPhil ${ }^{1}$ and \\ Muin J. Khoury, MD, PhD ${ }^{1,2}$
}

Purpose: The objective of this study was to identify trends and gaps in the field of implementation science in genomic medicine.

Methods: We conducted a literature review using the Centers for Disease Control and Prevention's Public Health Genomics Knowledge Base to examine the current literature in the field of implementation science in genomic medicine. We selected original research articles based on specific inclusion criteria and then abstracted information about study design, genomic medicine, and implementation outcomes. Data were aggregated, and trends and gaps in the literature were discussed.

Results: Our final review encompassed 283 articles published in 2014 , the majority of which described uptake $(35.7 \%, n=101)$ and preferences $(36.4 \%, n=103)$ regarding genomic technologies, particularly oncology $(35 \%, n=99)$. Key study design elements, such as racial/ethnic composition of study populations, were underreported in studies. Few studies incorporated implementation science theoretical frameworks, sustainability measures, or capacity building.

Conclusion: Although genomic discovery provides the potential for population health benefit, the current knowledge base around implementation to turn this promise into a reality is severely limited. Current gaps in the literature demonstrate a need to apply implementation science principles to genomic medicine in order to deliver on the promise of precision medicine.

Genet Med advance online publication 12 January 2017

Key Words: dissemination; genomic medicine; implementation; precision medicine; translational research

\section{INTRODUCTION}

In the past two and a half decades since the start of the Human Genome Project in 1990, researchers have advanced the field of genomics, making transformational discoveries with implications for improving human health. Genomic information is increasingly applied to disease diagnosis, management, and prevention. ${ }^{1}$ However, the speed of translation from bench to bedside to improve health has been relatively slow in relation to the speed of discovery. ${ }^{2}$ This may be partially due to gaps in the evidence base for clinical utility of genomic information and other barriers such as improving provider awareness and acceptability, as well as building infrastructure for genomic medicine delivery.

Genomic research can be considered within a framework of four translational phases of research in which the first phase after discovery is the characterization and development of candidate genomic health applications (T1). In the current scientific literature and federally funded grants, the majority of genomic research falls within the T0 and T1 phases. ${ }^{1,3,4}$ Phase 2-4 research (T2-4) attempts to translate genomic discovery into real-world settings and improved population health outcomes. More specifically, T2 research focuses on building an evidence base for clinical utility of genomic-based applications such as tests and interventions. T3 research examines how to move evidence-based genomic discoveries into health practice. Finally, T4 seeks to study efforts to scale up benefits of genomic discoveries to improve population health. Less than $2 \%$ of research falls within the T2-4 phases, demonstrating a need to increase research efforts in this area to implement promising genomic interventions into practice. ${ }^{5}$

Implementation science-the study and application of methods that promote uptake of research findings into practice-can address the challenges of genomic medicine. ${ }^{6}$ Implementation science can be incorporated into multiple translational phases of research to promote the integration of genomics into realworld practice settings. However, it remains unclear to what extent translational research in genomic medicine includes implementation science approaches. In this study, we aimed to identify the degree to which implementation science is present in genomic medicine research. We identified major gaps in the field that will be crucial to fill to ensure the population benefit of genomic medicine.

\section{MATERIALS AND METHODS}

We conducted a systematic literature review using the Centers for Disease Control and Prevention's Public Health Genomics Knowledge Base (PHGKB). ${ }^{7}$ Specifically, we used the Genomics and Health Impact Update component of this knowledge base. ${ }^{5}$

${ }^{1}$ Division of Cancer Control and Population Sciences, National Cancer Institute, Rockville, Maryland, USA; ${ }^{2}$ Office of Public Health Genomics, Centers for Disease Control and Prevention, Atlanta, Georgia, USA. Correspondence: Megan C. Roberts (megan.roberts@nih.gov) 
This database includes published literature identified through a PubMed search query and genomic-related Google Alerts and websites. ${ }^{5}$ Additional details regarding the CDC PHGKB and the impact updates have been previously published. ${ }^{7}$ The resulting articles were curated by two or more CDC staff members to identify published studies that fell within the T2-T4 phases in 2014 ( $n=626$ articles).

We developed inclusion and exclusion criteria (Table 1) and a codebook (Supplementary Table S1 online) using an iterative process. We included studies that contributed to our understanding of the implementation of genetic/-omic testing, genetic counseling, and collection of family history information. More specifically, the included studies focused on issues related to effectiveness, satisfaction, speed/timeliness, patientcenteredness (i.e., awareness/knowledge/attitudes/needs regarding genetic testing or counseling among stakeholders, including patients, public, providers), costs, uptake, recruitment, surveillance system development, and workforce issues. We excluded quality-assurance studies, content or policy analyses, predictive/prognostic model validations, efficacy studies of new technologies, risk-factor analyses, case studies, descriptive studies that only yielded the prevalence of a genetic disease or mutations, discovery or mechanism of action studies, healthservices research (unrelated to the actual implementation of genomic medicine) among high-risk populations, and studies reporting only psychosocial outcomes without any mention of practice or implementation. We also excluded articles that did not report research (articles with no methods and/or results sections), conference abstracts, and articles not written in English. One author (M.C.R.) reviewed abstracts and, when necessary, M.K. and D.C. resolved decisions for abstract inclusion. After abstracts had been assessed for inclusion/exclusion criteria, 484 full-text articles were reviewed.

The initial codebook was adapted from a National Institutes of Health portfolio review of Implementation Science-funded research. ${ }^{8}$ Terms related to genomic medicine were added and applied to a sample of 20 articles. The codebook was revised and then applied to all articles. To establish qualitative agreement between abstractors, $10 \%$ of articles were double-coded by two authors (M.C.R. and A.K.); when necessary, M.K. and D.C. resolved conflicting codes for articles. The remaining articles were coded by a single author (M.C.R. or A.K.).

Data were aggregated and summary statistics were calculated for the proportion of studies by code. We report study design, genomic medicine, and implementation outcomes. For implementation outcomes, we made a distinction between dissemination research (i.e., how information about genomic medicine is "packaged, transmitted, and interpreted among a variety of important stakeholder groups") and implementation research (efforts to integrate genomic medicine "within real-world public health, clinical and community service systems") to align with agency activities (e.g., funding opportunity announcements, conferences, training programs). ${ }^{9}$

\section{RESULTS}

Two hundred eighty-three of 626 articles were included in our final review (Supplementary Figure S1 online). Of the included articles, the vast majority (91.5\%) described T3 phase research and the minority described T2 $(4.2 \%, n=12)$ and T4 $(4.2 \%, n=12)$ research.

\section{Study design}

Most studies used quantitative $(80.6 \%, n=228)$ rather than qualitative methods $(13.1 \%, n=37)$. Seventeen studies incorporated mixed methods (6\%). Of the quantitative studies, a minority leveraged comparative effectiveness analyses $(5.7 \%, n=16)$ or only reported cost-effectiveness analyses $(1.4 \%, n=4)$. Race and ethnicity were severely underreported. More than half (59\%, $n=167)$ were missing all race/ethnicity data (i.e., white, black, Asian, Hispanic ethnicity data). Studies were more likely to be missing data regarding racial and ethnic minority populations than missing data regarding the proportion of white study participants. Because countries with homogeneous populations may be less likely to report race/ethnicity, we examined the proportion of US studies with missing data because the United States is a racially/ethnically diverse country and the majority of study populations consisted of US participants $(n=168)$. Although rates of missing data were lower in the United States, race and ethnicity remained underreported (44\% missing race/ ethnicity data). Overall, of the studies that reported race/ethnicity, the majority of study populations consisted of whites (mean, $76.8 \%$; median, $82.0 \%$ ), with a lower representation of blacks (mean, 24.1\%; median, 11.5), Asians (mean, 15.7; median, 4.5), and Hispanics (mean, 13.3; median, 5.9) (Figure 1).

Most studies were cross-sectional (68.9\%), with a minority of cohort studies $(9.5 \%, n=27)$ and pre-/post-studies $(8.8 \%$, $n=25)$. Rarely did studies use quasi-experimental trials $(1.1 \%$, $n=3)$, randomized control trials $(4.9 \%, n=14)$, or other designs (e.g., longitudinal studies that did not follow a particular cohort) $(6.7 \%, n=19)$. The majority of studies took place within a clinical setting $(65.4 \%, n=185)$, with only a few studies occurring within a public health setting $(24 \%, n=68)$, such as analyses through population registry-based registries or community-based studies. Finally, 30 studies (10.6\%) occurred within "other settings," such as direct-to-consumer studies and studies that occurred under the purview of another research study. Study sample sizes varied by study type (e.g., qualitative vs. quantitative; cross-sectional vs. randomized controlled trial), with a median of 221 participants (range, 10 to 1,504,535; mean, 16,821).

\section{Implementation outcomes}

Implementation research $(64.7 \%, n=183)$ primarily included studies that examined patients' and providers' attitudes and preferences about genetic testing or genetic counseling $(51.6 \%$, $n=146)$ and/or examined barriers and facilitators to genomic medicine $(18 \%, n=51)$. Approximately $19 \%$ of studies $(n=$ 
Table 1 Inclusion and exclusion criteria for literature review Inclusion criteria ${ }^{a}$

Broadly defined effectiveness studies $(n=133)$

Comparative effectiveness ( $n=16$ )

Patient satisfaction with genetic/genomic testing/counseling $(n=14)$

Efficiency/timeliness of genetic testing or counseling $(n=29)$

Patient/provider/public awareness/knowledge/attitudes/perceptions/needs regarding genetic testing $(n=141)$

Predictors of willingness to pay for genetic testing $(n=4)$

Uptake of testing and factors associated with uptake $(n=101)$

Strategies for recruitment into genetic/genomic research $(n=7)$

Development of surveillance systems $(n=2)$

Workforce $(n=3)$

\section{Exclusion criteria}

Content analysis of guidelines, policies, insurance criteria, literature reviews

Predictive/prognostic model validation or evaluation

Efficacy trial of a new test or method

Risk factor analysis

Case study

Article not written in English

Prevalence of mutations within a population

Discovery/mechanism of action

Conference abstract

Health services research among carriers or high-risk groups that does not include genetic testing/counseling/family history collection

Development of measures to assess psychosocial outcomes of mutation

carriers

Psychosocial outcomes only regarding genetic testing/counseling

Not a research study (no methods or results section)

Quality assessments without any implementation science components/ outcomes

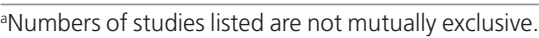

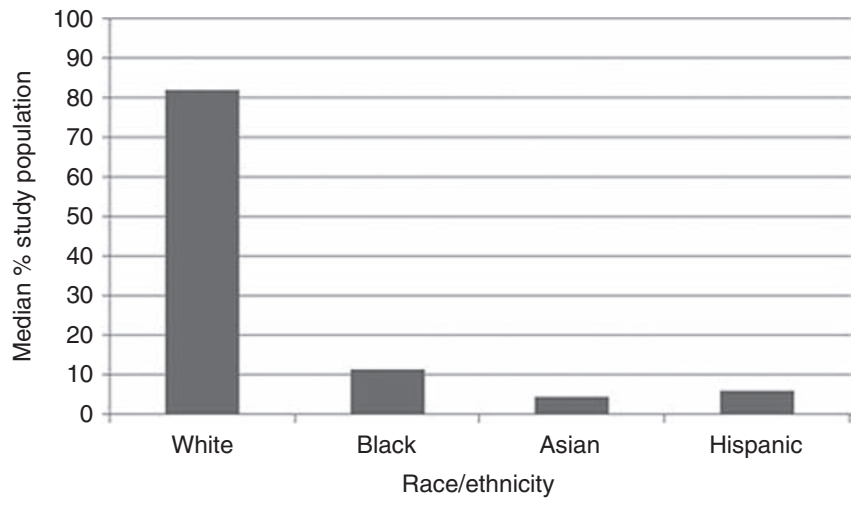

Figure 1 Median percent racial/ethnic composition of study populations in studies that reported race/ethnicity.

54) described dissemination research, which largely centered on preferences for genetic test communication or awareness of testing. Studies that described multiple types of research $(10.2 \%$, $n=29)$. Adoption-(3.9\%, $n=11)$, hybrid effectiveness- $(1.8 \%$, $n=5)$, and sustainability- $(0.4 \%, n=1)$ focused research was uncommon. Rarely were sustainability indicators (i.e., capacity
Notes

For the purposes of inclusion, this criterion includes effectiveness studies examining not only health outcomes but also clinical utility, uptake, costs, health outcomes of testing, and guideline adherence

Includes impact of media or advertising on awareness and includes providers' perspectives on readiness to deliver genetic services By insurers or patients Includes barriers and facilitators for uptake of genetic testing/ counseling building, maintenance, formal cost analysis) included in the studies $(7.1 \%, n=20)$; however, when included, indicators were typically formal cost analyses $(4.6 \%, n=13)$ rather than capacity building $(1.8 \%, n=5)$ or maintenance $(0.7 \%, n=2)$.

Only $1.8 \%$ of studies included implementation science frameworks $(n=5)$, with diffusion of innovations primarily used $(60 \%, n=3)$; typically, the frameworks were used in formative research $(60 \%, n=3)$ rather than for measurement $(20 \%, n=$ 1 ) or multiple purposes $(20 \%, n=1)$. RE-AIM was used in one study, and another study used a combination of implementation science frameworks. Collaborative processes were rarely $(1.8 \%, n=5)$ incorporated into the studies; if they were incorporated, then stakeholder engagement $(60 \%, n=3)$ was typically leveraged as opposed to team science approaches $(20 \%$, $n=1)$ or community-based participatory research $(20 \%, n=$ 1). Patient centeredness $(36.4 \%, n=103)$ and uptake $(35.7 \%$, $n=101)$ were the most commonly reported implementation outcomes (compared to effectiveness, acceptability, feasibility, costs, efficiency, satisfaction, timeliness, fidelity, inequity, and safety). Patient centeredness outcomes typically were operationalized as patient preferences or needs in relation to genetic testing or counseling. Of studies that reported costs associated 
with genetic testing, genetic counseling, or family history collection $(9.2 \%, n=26)$, the majority measured monetary costs $(57.7 \%, n=15)$ as opposed to nonmonetary costs $(11.5 \%, n=$ 3). Few studies used multiple measures of costs $(11.5 \%, n=3)$ or other measures of costs $(19.2 \%, n=5)$, such as willingness to pay. The majority of studies included individual-level data as the unit of analysis $(94 \%, n=266)$; only 16 studies used study site as the unit of analysis (5.7\%); for one study the unit of analysis was unclear.

\section{Genomic medicine outcomes}

Most studies $(90.4 \%, n=256)$ examined genetic/genomic testing or genetic counseling $(n=39)$, whereas only $9.5 \%$ of studies focused on family history collection $(n=27)$. In terms of the genetic/genomic technologies examined in the literature, the majority focused on germline testing $(67.5 \%, n=191)$ rather than somatic $(6.7 \%, n=19)$ or cell-free DNA $(3.5 \%, n=10)$ testing. A minority of studies examined genomic medicine more broadly, examined genetic counseling or family history collection exclusively, or examined a combination of germline, somatic, and cell-free DNA testing $(22.3 \%, n=63)$. All tests were genetics/genomics-based and included gene sequencing, single-nucleotide polymorphism genotyping, whole-genome or whole-exome sequencing, gene expression analyses, and/ or chromosomal analyses, with the exception of newborn screening, which often also included biomarker tests. No other "-omic" studies (e.g., metabolomics) were represented in the implementation science literature that we reviewed.

Most genomic medicine tools were used for risk assessment $(36.4 \%, n=103)$ or diagnostics $(30.4 \%, n=86)$. A minority of the tests were therapeutic $(12.7 \%, n=36)$, including pharmacogenomic tests as well as prognostic tests that were applied to aid treatment decision-making. Among applicable studies $(n=$ 266), the clinical applications of these tests were overwhelmingly used for cancer prevention or treatment $(37.2 \%, n=$ 99), with prenatal testing $(9.5 \%, n=27)$ and newborn screening $(8.5 \%, n=24)$ being the next most common contexts for genomic medicine (Figure 2). Other clinical applications

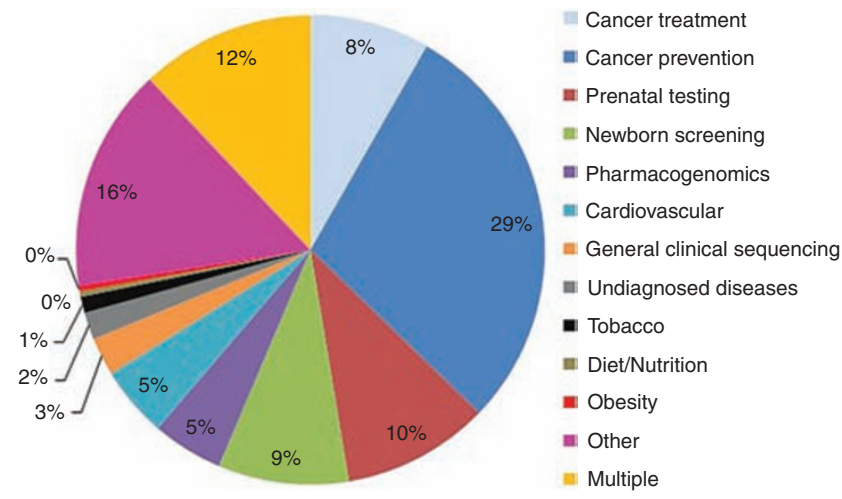

Figure 2 Clinical applications of genetic/genomic tests examined in studies for which this field was applicable. The majority of "multiple" included tests for which there were both cancer treatment and cancer prevention applications. included diabetes, Alzheimer disease, autism, other mental illness, Parkinson disease, infertility, relatedness, and retinal disease. For cancer, BRCA1/2 testing and Lynch syndrome were among the most common tests described in the literature.

\section{DISCUSSION}

Examining trends in the literature, the number of articles spanning T2-T4 research in genomic medicine increased from 505 articles in 2012 to 626 articles in $2014 .{ }^{5}$ The majority of included studies reflected $\mathrm{T} 3$ phase research assessing the implementation of genomic medicine into clinical practices, with few T4 research studies demonstrating that research documenting the population health impact of genomic medicine implementation is severely lacking. To address this issue, Chambers et al. ${ }^{6}$ recently proposed a new framework for conducting research and documenting improvements in health outcomes in realworld practice settings, specifically, the intersection of precision medicine, implementation science, and learning health-care systems (i.e., health-care systems that undergo continuous improvement through data capture that is leveraged for healthcare improvements). Within this framework, implementation science approaches can aid the translation of genomic medicine into practice in an iterative fashion that leverages the learning health-care system as a platform. Although such a framework holds promise, the current literature uncovered major gaps that prevent making such a framework a reality.

\section{Models and context}

Very few studies incorporated implementation science theoretical models to underpin their research. Perhaps as a result, many studies lacked fundamental components relevant to implementation science. For example, many theoretical frameworks account for multilevel factors that influence the implementation of genomic technology. However, the majority of the reviewed literature focused on individual-level factors associated with implementation of genomic medicine rather than macro-level factors that influence the adoption of genomic medicine by health-care systems or clinics. Furthermore, the reviewed studies often did not thoroughly describe or measure key contextual variables, such as characteristics of the clinical settings, making it difficult to conceptualize the generalizability of study findings. Moving forward, genomic research should incorporate rigorous implementation science methods grounded in theory and describe contextual factors.

\section{Real-world settings}

Another key component of implementation science is to examine genomic medicine implementation within real-world settings beyond the academic medical center and in diverse and representative populations. The majority of studies did not include data regarding the racial/ethnic composition of their study populations. This may be partially due to the current International Committee of Medical Journal Editors guidelines for the reporting of race/ethnicity in publications, which recommends the reporting of race/ethnicity data when they 
are available and relevant to the study. ${ }^{10}$ Furthermore, quality of race/ethnicity data should be considered, as well as the justification for the use and operationalization of race/ethnicity constructs. ${ }^{10,11}$ As such, some of the underreporting that we captured may be justifiable within individual study settings. However, overall underreporting race/ethnicity data may be problematic because lack of reporting prevents the ability to monitor equitable uptake and implementation of these innovations across diverse populations. ${ }^{11}$ Of the studies that did report racial/ethnic status, the study populations consisted primarily of whites, demonstrating the need to incorporate underserved and minority participants into genomic medicine research to prevent exacerbating existing disparities in health-care quality and access. ${ }^{12-14}$

In addition, our review found that genomic medicine studies that intersect with implementation science did not span a broad range of clinical and public health contexts. Most of the reviewed studies occurred within the cancer and risk assessment contexts, which more broadly reflect similar trends in genomic medicine research. ${ }^{5}$ This is probably due to the fact that current Healthy People 2020 objectives for genomics are available only for cancer-related testing (i.e., BRCA1/2 and Lynch syndrome testing). ${ }^{15} \mathrm{As}$ such, there remains a need to move beyond implementation of genomic medicine in oncology to other disease applications and to examine not only genetic counseling and genomics but other-omics tools as well.

\section{Evidence-based implementation strategies}

Evidence-based implementation strategies will be necessary for the field to move forward. The current literature is primarily descriptive, using observational data and cross-sectional or pooled cross-sectional data analyses. Many studies have documented uptake of testing, barriers and facilitators to implementation, and the attitudes, preferences, and knowledge of key stakeholders. As such, it is time to move beyond cross-sectional study designs and into intervention work for improving implementation of genomic medicine that has a rich observational evidence base and demonstrated clinical utility. This can be accomplished by incorporating the current observational evidence in genomic medicine with evidence-based implementation strategies, recently organized into key domains: planning, educating, financing, restructuring, managing quality, and attending to the policy context. ${ }^{16}$

\section{Collaborative processes}

Finally, among intervention studies, few reported using collaborative processes in their study design. Incorporating patients, providers, administrators, researchers, policy makers, and community leaders throughout the research process joins multilevel processes that support equitable implementation of genomic medicine. These approaches, along with explicitly addressing issues of sustainability, will be important moving forward. Although a small number of studies examined monetary costs, few studies examined nonmonetary costs or focused on building infrastructure or the workforce necessary to deliver genomic medicine within the clinical and public health settings.

\section{Limitations}

Although we used a validated database to identify relevant articles for our review, it is possible that relevant articles were unintentionally excluded from our search. In addition, we used broad inclusion criteria, at the cost of specificity, in an effort to gain a better understanding of the current body of literature that focuses on implementation of genomic medicine. As such, we may have included articles that would not traditionally fall under the umbrella of implementation science. In some cases, articles had missing information about key components within the study design, relevant to our codebook. When possible, we made inferences; however, it is possible that codes were underreported or misclassified as a result. Finally, we did not examine national funding opportunities for implementation research for the included studies. Future studies should examine the current funding landscape for such research and explore the role that funding can play in bridging gaps in the literature.

\section{Conclusions}

For the field of genomic medicine to succeed, a more robust implementation science agenda and tool development are needed. Multidisciplinary stakeholders, including genomics researchers, clinical practitioners, and health services researchers, should play a role in shaping research priorities and funding opportunities to advance this agenda. Current National Institutes of Health (NIH) initiatives may help move the field forward and close the gaps identified in the current literature. $\mathrm{NIH}$ initiatives such as the Implementing Genomics in Practice (IGNITE) consortium ${ }^{17,18}$ and Clinical Sequencing Exploratory Research (CSER) program ${ }^{19,20}$ support the incorporation of genomic data into clinical care in diverse clinical settings. Furthermore, funding announcements for dissemination and implementation research now specifically mention genomic medicine, ${ }^{9}$ and the $\mathrm{NIH}$ requires the collection of information regarding race and ethnicity for studies involving primary data collection for all human subjects research. Finally, the NIH Precision Medicine Initiative and its recruitment of a 1-millionperson cohort will allow the opportunity for discovery science (knowledge generation) with short-term implementation. ${ }^{21}$ However, although recent NIH initiatives may help close the glaring gaps identified in this review, much more is needed.

This review identified several opportunities to advance the field of genomic medicine implementation research. First, increased attention should be given to the application of evidence-based genomic medicine in real-world settings with diverse patient populations in order to optimize impact and prevent health inequities. Furthermore, the incorporation of evidence-based implementation strategies and frameworks would improve the rigor in which genomic medicine implementation science is conducted. By incorporating these principles into genomics research, we can more effectively move 
evidence-based discoveries to the clinic. Now is the time to design the next generation of genomic studies across the spectrum of diseases and life stages that incorporate principles of implementation science into their design and conduct.

\section{SUPPLEMENTARY MATERIAL}

Supplementary material is linked to the online version of the paper at http://www.nature.com/gim

\section{ACKNOWLEDGEMENTS}

The findings and conclusions in this report are those of the authors and do not necessarily reflect the views of the Department of Health and Human Services.

\section{DISCLOSURE}

The authors declare no conflict of interest.

\section{REFERENCES}

1. Khoury MJ, Gwinn M, Yoon PW, Dowling N, Moore CA, Bradley L. The continuum of translation research in genomic medicine: how can we accelerate the appropriate integration of human genome discoveries into health care and disease prevention? Genet Med 2007;9:665-674.

2. Manolio TA, Chisholm RL, Ozenberger $B$, et al. Implementing genomic medicine in the clinic: the future is here. Genet Med 2013;15:258-267.

3. Schully SD, Benedicto CB, Gillanders EM, Wang SS, Khoury MJ. Translational research in cancer genetics: the road less traveled. Public Health Genomics 2011:14:1-8.

4. Schully SD, Khoury MJ. What is translational genomics? An expanded research agenda for improving individual and population health. Appl Trans/ Genom 2014;3:82-83

5. Clyne M, Schully SD, Dotson WD, et al. Horizon scanning for translational genomic research beyond bench to bedside. Genet Med 2014;16:535-538.

6. Chambers DA, Feero WG, Khoury MJ. Convergence of implementation science, precision medicine, and the learning health care system: a new model for biomedical research. JAMA 2016;315:1941-1942.
7. YuW, Gwinn M, Dotson WD, et al. A knowledge base for tracking the impact of genomics on population health. Genet Med 2016;18:1312-1314.

8. Neta G, Sanchez MA, Chambers DA, et al. Implementation science in cancer prevention and control: a decade of grant funding by the National Cancer Institute and future directions. Implement Sci 2015;10:4.

9. US Department of Health and Human Services. Grants and Funding. 2016. https://grants.nih.gov/grants/guide/pa-files/PAR-16-238.html. Accessed 1 July 2016.

10. International Committee of Medical Journal Editors. Recommendations for the Conduct, Reporting, Editing, and Publication of Scholarly Work in Medical Journals. 2016. http://www.icmje.org/icmje-recommendations.pdf. Accessed 23 September 2016.

11. Ma IW, Khan NA, Kang A, Zalunardo N, Palepu A. Systematic review identified suboptimal reporting and use of race/ethnicity in general medical journals. J Clin Epidemio/ 2007;60:572-578.

12. Spratt $D E$, Chan $T$, Waldron $L$, et al. Racial/ethnic disparities in genomic sequencing. JAMA Oncol 2016;2:1070-1074.

13. Need AC, Goldstein DB. Next generation disparities in human genomics: concerns and remedies. Trends Genet 2009;25:489-494.

14. Manrai AK, Funke BH, Rehm HL, et al. Genetic misdiagnoses and the potential for health disparities. N Eng/ J Med 2016;375:655-665.

15. Office of Disease Prevention and Health Promotion. 2020 Topics \& Objectives: Genomics. 2016. https://www. healthypeople.gov/2020/topics-objectives/topid genomics/objectives. Accessed 23 September 2016.

16. Powell BJ, Waltz TJ, Chinman MJ, et al. A refined compilation of implementation strategies: results from the Expert Recommendations for Implementing Change (ERIC) project. Implement Sci 2015;10:21.

17. Weitzel KW, Alexander M, Bernhardt BA, et al.; IGNITE Network. The IGNITE network: a model for genomic medicine implementation and research. BMC Med Genomics 2016;9:1.

18. National Human Genome Research Institute. Implementing Genomics in Practice (IGNITE). 2016. https://www.genome.gov/27554264/implementinggenomics-in-practice-ignite/. Accessed 1 July 2016.

19. Green RC, Goddard KA, Jarvik GP, et al.; CSER Consortium. Clinical Sequencing Exploratory Research Consortium: accelerating evidence-based practice of genomic medicine. Am J Hum Genet 2016;98:1051-1066.

20. CSER Consortium. 2016. https://cser-consortium.org/. Accessed 1 July 2016

21. Khoury MJ, Evans JP. A public health perspective on a national precision medicine cohort: balancing long-term knowledge generation with early health benefit. JAMA 2015;313:2117-2118. 